\title{
Phonon blockade in a nanomechanical resonator resonantly coupled to a qubit
}

\author{
Xun-Wei Xu, ${ }^{1, *}$ Ai-Xi Chen,,${ }^{1} \dagger$ and Yu-xi Liu ${ }^{2,3}$ \\ ${ }^{1}$ Department of Applied Physics, East China Jiaotong University, Nanchang, 330013, China \\ ${ }^{2}$ Institute of Microelectronics, Tsinghua University, Beijing 100084, China \\ ${ }^{3}$ Tsinghua National Laboratory for Information Science and Technology (TNList), Beijing 100084, China
}

(Dated: September 18, 2018)

\begin{abstract}
We study phonon statistics in a nanomechanical resonator (NAMR) which is resonantly coupled to a qubit. We find that there are two different mechanisms for phonon blockade in such a resonantly coupled NAMR-qubit system. One is due to the strong anharmonicity of the NAMR-qubit system with large coupling strength; the other one is due to the destructive interference between different paths for two-phonon excitation in the NAMRqubit system with a moderate coupling strength. We find that the phonon blockade is fragile towards thermal mode occupations and can only be observed for NAMR being at ultracold effective temperature. In order to enlarge the mean phonon number for strong phonon antibunching with a moderate NAMR-qubit coupling strength, we assume that two external driving fields are applied to the NAMR and qubit, respectively. In this case, we find that the phonon blockades under two mechanisms can appear at the same frequency regime by optimizing the strength ratio and phase difference of the two external driving fields.
\end{abstract}

PACS numbers: 42.50.Pq, 42.50.Ar, 85.85.+j, 85.25.-j

\section{INTRODUCTION}

Quantum effects in a nanomechanical resonator (NAMR) can be explored when the vibration energy of NAMR can beat the thermal energy and approach the quantum limit. Recently, the experiment showed that the quantum limit has been reached in a NAMR with a sufficiently high frequency at a very low temperature [1]. This makes it possible for NAMR to be applied to quantum information processing [2].

If a NAMR approaches the quantum regime and the quanta of the mechanical oscillation, referred to as phonons, can be generated one by one, then a purely quantum phenomenon, phonon blockade, can be explored. In analogy to the Coulomb blockade [3] and photon blockade [4], the phonon blockade [5] is a phenomenon that only one phonon can be excited in a nonlinear mechanical oscillator [6] by external driving fields. Phonon blockade has already been studied in a NAMR coupled to a superconducting qubit in the dispersive regime [5, 7-9]. It should be more easily observed for larger nonlinear phonon interaction induced by the qubit, which corresponds to a larger coupling strength and moderate detuning between the NAMR and the qubit [5].

In contrast to Refs. [5, 7-9], where the NAMR is dispersively coupled to the qubit, here we are going to study the phonon statistics in a NAMR which is resonantly coupled to a qubit. In recent several works, a similar system, in which a single two-level defect system is coupled to an optomechanical system $[10,11]$, was proposed. Such a system can be used to realize phonon blockade due to the strong anharmonicity of the eigenstates corresponding to large NAMR-qubit coupling strength [10] in analogy to cavity QED [12-15], and we call it as conventional phonon blockade (CPNB). Different from the previous studies [10], we here show that phonon blockade in

\footnotetext{
*Electronic address: davidxu0816@163.com

${ }^{\dagger}$ Electronic address: aixichen@ecjtu.edu.cn
}

the resonantly coupled NAMR-qubit system can also be produced by the destructive interference between different paths for two-phonon excitation with a moderate NAMR-qubit coupling strength. We call the interference-based phonon blockade as unconventional phonon blockade (UCPNB), which is similar to the unconventional photon blockade in a weakly nonlinear system of photonic molecule [16-27]. Moreover, we find that the phonon blockade is fragile towards thermal mode occupations for the small mean phonon number and can only be observed for NAMR being at ultracold effective temperature.

To improve the robustness of the phonon blockade against the thermal noise and to increase the number of single phonons generated in a given time, we discuss how to enlarge the mean phonon number for strong phonon antibunching with a moderate NAMR-qubit coupling. Recently, photon blockade effect in a quantum dot-cavity system for the cavity and the quantum dot driven by two external fields respectively was studied [28], and an enhancement of photon blockade with large photon number was achieved with some optimized parameters for the two driving fields. In the spirit of the approach of Ref. [28], we assume that two external driving fields are applied to the NAMR and qubit, respectively, then a large mean phonon number for strong phonon antibunching is obtained with a moderate NAMR-qubit coupling strength. The optimal detuning for CPNB is mainly determined by the NAMR-qubit coupling strength, while the optimal detuning for UCPNB is related to the strength ratio and phase difference of the two external driving fields. A large mean phonon number for strong phonon antibunching with a moderate NAMRqubit coupling strength can be obtained by combining the effects of CPNB and UCPNB when they appear at the same frequency regime by optimizing the strength ratio and phase difference of the two external driving fields.

The paper is organized as follows. In Sec. II, we describe the theoretical model of the resonant coupling between a NAMR and a qubit, and then we give analytical results of the optimal conditions for observing phonon blockade. In Sec. III, 
we study the phonon blockade when the external driving field is not applied to the qubit. In Sec. IV, we consider how to enlarge the mean phonon number for the phonon blockade by an external driving field applied to the qubit. In Sec. V, we study how to detect the phonon blockade by an optical cavity which is optomechanically coupled to the NAMR. Finally, the main results of our work are summarized in Sec. VI.

\section{THEORETICAL MODEL AND ANALYTICAL RESULTS}

Motivated by a recent experiment [1], we study the phonon blockade in a quantum system, in which a high-frequency NAMR is coupled to a phase qubit, as schematically shown in Fig. 1. The Hamiltonian of the whole system is given by $(\hbar=1)$

$$
\begin{aligned}
H_{\mathrm{mq}}= & \omega_{0} \sigma_{+} \sigma_{-}+\omega_{m} b^{\dagger} b+J\left(\sigma_{+} b+b^{\dagger} \sigma_{-}\right) \\
& +\left(\Omega e^{-i \phi} e^{-i \omega_{q} t} \sigma_{+}+\varepsilon b^{\dagger} e^{-i \omega_{b} t}+\text { H.c. }\right)
\end{aligned}
$$

under the rotating wave approximation, where $\sigma_{+}$and $\sigma_{-}$ are the raising and lowering operators of the qubit with frequency $\omega_{0} ; b$ and $b^{\dagger}$ denote the annihilation and creation operators of the NAMR with frequency $\omega_{m} ; J$ is the NAMR-qubit coupling strength; $\Omega(\varepsilon)$ describes the coupling strength between tha qubit (NAMR) and the external driving field with frequency $\omega_{q}\left(\omega_{b}\right)$ and $\phi$ is the phase difference between the two external driving fields. Hereafter we assume that $\Omega$ and $\varepsilon$ are real numbers and the frequencies of the two driving fields are the same and $\omega_{q}=\omega_{b}=\omega_{d}$. In this paper, we focus on the phonon blockade for the resonant NAMR-qubit coupling, i.e., $\omega_{0}=\omega_{m}$. In the rotating reference frame with the frequency $\omega_{d}$ of the driving fields, the Hamiltonian of the quantum system is shown as

$$
\begin{aligned}
H_{\mathrm{mq}}^{\prime}= & \Delta \sigma_{+} \sigma_{-}+\Delta b^{\dagger} b+J\left(\sigma_{+} b+b^{\dagger} \sigma_{-}\right) \\
& +\left(\Omega e^{-i \phi} \sigma_{+}+\varepsilon b^{\dagger}+\text { H.c. }\right)
\end{aligned}
$$

with detuning $\Delta \equiv \omega_{0}-\omega_{d}=\omega_{m}-\omega_{d}$.

The second-order correlation function of the phonons in the steady state (i.e., $t \rightarrow+\infty$ ) is defined by

$$
g_{b}^{(2)}(\tau) \equiv \frac{\left\langle b^{\dagger}(t) b^{\dagger}(t+\tau) b(t+\tau) b(t)\right\rangle}{n_{b}^{2}}
$$

where $n_{b} \equiv\left\langle b^{\dagger}(t) b(t)\right\rangle$ is the mean phonon number in the NAMR. The correlation function of the phonons can be calculated by numerically solving the master equation for the density matrix $\rho$ of the system [29]

$$
\begin{aligned}
\frac{\partial \rho}{\partial t}= & -i\left[H_{\mathrm{mq}}^{\prime}, \rho\right] \\
& +\gamma\left(n_{m, \mathrm{th}}+1\right) L[b] \rho+\gamma n_{m, \mathrm{th}} L\left[b^{\dagger}\right] \rho \\
& +\kappa\left(n_{q, \mathrm{th}}+1\right) L\left[\sigma_{-}\right] \rho+\kappa n_{q, \mathrm{th}} L\left[\sigma_{+}\right] \rho,
\end{aligned}
$$

where $L[o] \rho=o \rho o^{\dagger}-\left(o^{\dagger} o \rho+\rho o^{\dagger} o\right) / 2$ denotes a Lindbland term for an operator $o, \kappa$ is damping rate of the qubit

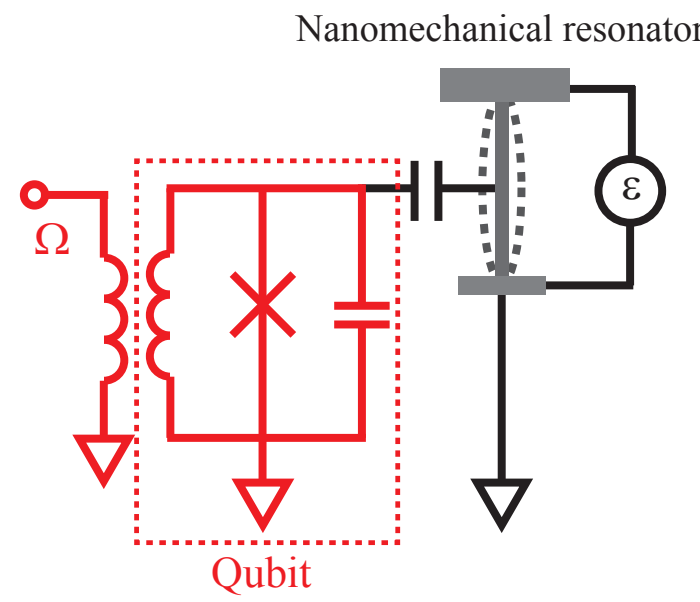

FIG. 1: (Color online) Schematic diagram that a nanomechanical resonator (gray) is coupled to a phase qubit (red). The nanomechanical resonator and phase qubit are driven by external fields with strengths $\varepsilon$ and $\Omega$, respectively.

and $\gamma$ is damping rate of the NAMR; $n_{q, \text { th }}$ and $n_{m \text {,th }}$ are the mean numbers of the thermal photon (phonon), given by the Bose-Einstein statistics $n_{q, \text { th }}=\left[\exp \left(\hbar \omega_{0} / k_{B} T\right)-1\right]^{-1}$, $n_{m, \mathrm{th}}=\left[\exp \left(\hbar \omega_{m} / k_{B} T\right)-1\right]^{-1} . k_{B}$ is the Boltzmann constant and $T$ is the temperature of the reservoir at the thermal equilibrium. We assume that $n_{\mathrm{th}} \equiv n_{q, \mathrm{th}}=n_{m \text {, th }}$ for the resonant condition $\omega_{0}=\omega_{m}$.

Before the numerical calculations of the correlation function of the phonons, it is instructive to find the optimal conditions for strong phonon antibunching. It has been shown in Ref. [10] that one can observe the phonon antibunching (CPNB) at $\Delta= \pm J$ with strong NAMR-qubit coupling $(J>\kappa, \gamma)$ at a low temperature.

Under the weak driving condition $\{\Omega, \varepsilon\}<\{\kappa, \gamma\}$, the optimal condition for UCPNB can be derived analytically following the approach given in Ref. [17]. The optimal condition for UCPNB, in the limit $T \rightarrow 0$, is a second-order equation in the strength ratio $\eta \equiv \Omega / \varepsilon$ as

$$
A_{2} \eta^{2} e^{-i 2 \phi}+A_{1} \eta e^{-i \phi}+A_{0}=0
$$

with the coefficients $A_{i}(i=0,1,2)$ defined by

$$
\begin{aligned}
& A_{2} \equiv \sqrt{2} J_{\mathrm{opt}}^{2} \\
& A_{1} \equiv-2 \sqrt{2} J_{\mathrm{opt}}\left(2 \Delta_{\mathrm{opt}}-i \frac{\kappa+\gamma}{2}\right) \\
& A_{0} \equiv \sqrt{2} J_{\mathrm{opt}}^{2}+\sqrt{2}\left(\Delta_{\mathrm{opt}}-i \frac{\kappa}{2}\right)\left(2 \Delta_{\mathrm{opt}}-i \frac{\kappa+\gamma}{2}\right)(8)
\end{aligned}
$$

where $\Delta_{\text {opt }}$ and $J_{\text {opt }}$ are the optimal parameters for phonon blockade (i.e., $g_{b}^{(2)}(0) \rightarrow 0$ ) with the corresponding parameters of the external driving fields $(\varepsilon, \Omega$ and $\phi)$. The details of the derivation for Eq. (5) are given in Appendix A.

If the microwave driving field is not applied to the qubit (i.e., $\Omega=0$ ), Eq. (5) becomes

$$
A_{0}=0
$$


and the optimal conditions are written as

$$
\begin{aligned}
\Delta_{\mathrm{opt}} & =0, \\
J_{\mathrm{opt}} & =\frac{1}{2} \sqrt{\kappa(\kappa+\gamma)} .
\end{aligned}
$$

So if the microwave driving field is not applied to the qubit, the parameters $\Delta_{\text {opt }}$ and $J_{\text {opt }}$ for phonon blockade are determined by the decay rates ( $\kappa$ and $\gamma$ ) of the qubit and NAMR. For the case that a microwave driving field is applied to the qubit (i.e., $\Omega \neq 0$ ), the two solutions of Eq. (5) are given by

$$
\begin{aligned}
\eta_{ \pm} e^{-i \phi_{ \pm}} & =\left(\frac{2 \Delta_{\mathrm{opt}}}{J_{\mathrm{opt}}}-i \frac{\kappa+\gamma}{2 J_{\mathrm{opt}}}\right) \\
& \pm \sqrt{\left(\frac{\Delta_{\mathrm{opt}}}{J_{\mathrm{opt}}}-i \frac{\gamma}{2 J_{\mathrm{opt}}}\right)\left(\frac{2 \Delta_{\mathrm{opt}}}{J_{\mathrm{opt}}}-i \frac{\kappa+\gamma}{2 J_{\mathrm{opt}}}\right)-1}
\end{aligned}
$$

This implies that we can choose the parameters $\Delta_{\mathrm{opt}}$ and $J_{\mathrm{opt}}$ for phonon blockade, and the corresponding parameters for the two external driving fields $(\varepsilon, \Omega$ and $\phi)$ are determined by Eq. (12).

\section{PHONON BLOCKADE WITHOUT DRIVING FIELD APPLYING ON THE QUBIT $(\Omega=0)$}

We now study the phonon statistics via the second-order correlation function $g_{b}^{(2)}(\tau)$ and the mean phonon number $n_{b}=\left\langle b^{\dagger}(t) b(t)\right\rangle$ when the driving field is not applied to the qubit (i.e., $\Omega=0$ ). They are calculated numerically by solving the master equation [Eq. (4)] within a truncated Fock space $[8,16]$. The effect of a microwave driving field applied to the qubit (i.e., $\Omega \neq 0$ ) will be discussed in the next section.

In Fig. 2, the equal-time second-order correlation function $g_{b}^{(2)}(0)$ is plotted as a function of the rescaled detuning $\Delta / \kappa$ and the rescaled coupling strength $J / \kappa$. Phonon blockade appears in two different parameter regions: (i) $\Delta= \pm J$ for $J>\kappa$, i.e., the blue (dark) region along with the red dash lines in Fig. 2(a); (ii) the blue (dark) region around the point ( $\Delta=0, J=\kappa / \sqrt{2} \approx 0.71 \kappa$ ), as shown in Fig. 2(b).

Phonon blockade appearing in two different parameter regions can be interpreted in two different ways. Phonon blockade appearing in the region (i) ( $\Delta= \pm J$ for $J>\kappa$ ) results from the anharmonicity of the eigenstates of the strong coupling NAMR-qubit system. The energy-level diagram of one mechanical mode strongly coupled to a qubit is shown in Fig. 2(c), where $|n, \pm\rangle$ are the eigenstates (dressed states) of a mechanical mode (with the phonon number states $|n\rangle$ ) coupled to a qubit (with two states $|g\rangle$ and $|e\rangle$ ). The phonon, absorbed resonantly by the transition from $|0, g\rangle$ to $|1, \pm\rangle$, blocks the transition from $|1, g\rangle$ to $|2, \pm\rangle$ for large detuning. This kind of phonon blockade can be called as conventional phonon blockade (CPNB) in analogy with the phenomena of photon blockade in cavity QED [12-15].

Phonon blockade appearing in the region (ii) with weak coupling strength $(J<\kappa)$ can be explained using the destructive interference between two different paths of twophonon excitation as illustrated in Fig. 2(d) for transition
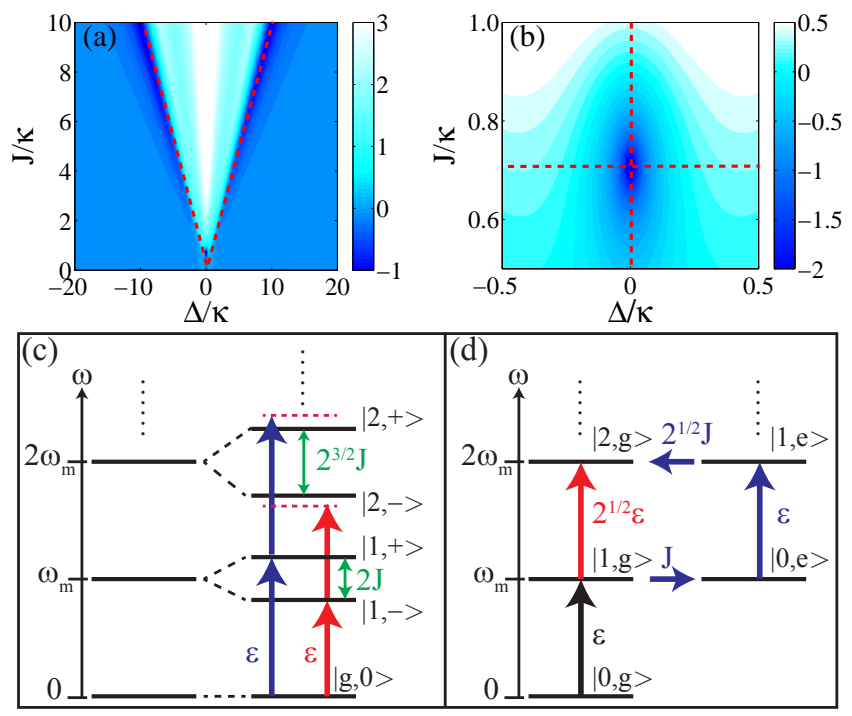

FIG. 2: (Color online) (a) Logarithmic plot (of base 10) of the equaltime second-order correlation function $\log _{10} g_{b}^{(2)}(0)$ as a function of the detuning $\Delta / \kappa$ and the linear coupling strength $J / \kappa$ for the parameters: $\gamma=\kappa, \varepsilon=0.01 \kappa, \Omega=0$ and $n_{\mathrm{th}}=0$. (b) shows a magnification of (a) at small $\Delta$ and $J$. (c) Energy-level diagram of one mechanical mode strongly coupled to a qubit. (d) Transition paths leading to the quantum interference responsible for the strong phonon antibunching.

paths $|1, g\rangle \rightarrow|2, g\rangle$ and $|1, g\rangle \rightarrow|0, e\rangle \rightarrow|1, e\rangle \rightarrow$ $|2, g\rangle$. We call the interference-based phonon blockade appearing in the region (ii) as unconventional phonon blockade (UCPNB). It is worth mentioning that the strong photon antibunching based on quantum interference has been found in the weakly nonlinear photonic molecules [16-27]. However, their is difference between our study here and that of nonlinear photonic molecules. The main difference is that there are two separate energy scales in the weakly nonlinear photonic molecules [16-27], one is large linear coupling strength between coupled cavity modes, and second is small nonlinearity (up to hundred times smaller than the photon damping rate). In the NAMR-qubit system, there is only a single energy parameter $J$, i.e., the NAMR-qubit coupling strength, which is reminiscent to the tunneling rate in photonic systems and is the order of the damping rates of the qubit or NAMR [Eq. (11)], while a large nonlinearity is hidden in the qubit structure.

The conditions for the appearance of phonon blockade in region (ii) can be understand by the following way. For $\Delta=0$, the amplitude for transition $|1, g\rangle \rightarrow|2, g\rangle$ is proportional to $\sqrt{2} \varepsilon$ and the amplitude for transition $|1, g\rangle \rightarrow$ $|0, e\rangle \rightarrow|1, e\rangle \rightarrow|2, g\rangle$ is proportional to $4 \sqrt{2} \varepsilon J^{2} /[\kappa(\kappa+\gamma)]$, where $\kappa / 2$ and $(\kappa+\gamma) / 2$ are the decay rates of the states $|0, e\rangle$ and $|1, e\rangle$, respectively. The perfect destructive quantum interference for strong phonon antibunching is only achieved in the condition that the transition matrix elements of these two paths of phonon excitation have the same amplitude but inverse phase, thus we obtain the optimal condition $J_{\text {opt }} \propto$ $\sqrt{\kappa(\kappa+\gamma)} / 2$. This is consistent well with the analytical re- 
sult given in Eqs. (11).

In order to see these more clearly, a few snapshots taken from Figs. 2(a) and 2(b) are shown in Fig. 3. The equaltime second-order correlation functions $g_{b}^{(2)}(0)$ are plotted as functions of the rescaled detuning $\Delta / \kappa$ in Figs. 3(a) and 3(b) and plotted as the function of the rescaled coupling strength $J / \kappa$ in Figs. 3(c) and 3(d). As shown in Fig. 3(a) for CPNB, with a strong NAMR-qubit coupling strength $J=10 \kappa$, we can obtain $g_{b}^{(2)}(0) \approx 0.1$ at $\Delta= \pm J$, and CPNB should be more easily observed for larger NAMR-qubit coupling strength $J$ [see, Fig. 3(c)]. As illustrated in Figs. 3(b) and $3(\mathrm{~d})$, the minimal value of the second-order correlation function $g_{b}^{(2)}(0) \approx 0.003$ can be reached with a weak NAMRqubit coupling strength $(J=\kappa / \sqrt{2})$ at $\Delta=0$ [predicted by Eqs. (10) and (11) for UCPNB]. The second-order correlation function $g_{b}^{(2)}(\tau)$ is plotted as a function of the normalized time delay $\tau /(2 \pi / \kappa)$ in Fig. 3(e) for $J=10 \kappa$ (CPNB) and $|\Delta| / \kappa$ taking the values 10, 9.2, 11 and in Fig. 3(f) for $J / \kappa=0.71$ (UCPNB) and $\Delta / \kappa$ taking the values $0,0.1,0.2$. The time duration for both CPNB and UCPNB is of the order of the life time of the phonons in the NAMR.

Figures 4(a) and 4(b) display the equal-time second-order correlation function $\log _{10} g_{b}^{(2)}(0)$ as functions of $\gamma / \kappa$ and $J / \kappa$ for $\Delta=10 \kappa$ in Fig. 4(a) and $\Delta=0$ in Fig. 4(b). $\log _{10} g_{b}^{(2)}(0)$ as a function of $J / \kappa$ with different $\gamma / \kappa$ is shown in Fig. 4(c) for $\Delta=10 \kappa$ and in Fig. 4(d) for $\Delta=0$. With increasing $\gamma / \kappa$, CPNB at $\Delta=10 \kappa$ has been gradually weakened [see, Figs. 4(a) and 4(c)] and a larger value of $J / \kappa$ is need for the same value of $g_{b}^{(2)}(0)$. Similarly, the optimal value of $J / \kappa$ for UCPNB increases with $\gamma / \kappa$ [see, Figs. 4(b) and 4(d)]. The optimal value of $J / \kappa$ for UCPNB [blue dark regime in Fig. 4(b)] is agree well with the analytical result given in Eq. (11) [yellow dash curve in Fig. 4(b)]. For simplicity of discussions, we choose $\gamma=\kappa$ in the following. However, we should mention that different ratios $\gamma / \kappa$ corresponded to different optimal conditions for UCPNB.

Different from the study on photon blockade in cavity QED or nonlinear photonic molecules, where the thermal photon effects can be neglected safely, because the mean thermal photon number for an optical cavity is negligible small, e.g., the thermal photon number for an optical cavity with frequency $3.21 \times 10^{14} \mathrm{~Hz}$ in Ref. [12] is smaller than $10^{-22}$ at the room temperature. While the effect of the thermal phonons should be considered in the observation of phonon blockade even with microwave-frequency nanomechanical resonators. Because the mean thermal phonon number becomes about $10^{-5}$ for a mechanical resonator with resonance frequency of $6 \mathrm{GHz}$ at a temperature of $25 \mathrm{mK}$ [1] and we will find that even this small mean thermal phonon number may have significant effect on the observation of phonon blockade.

We present in Figs. 5(a) and 5(b) the equal-time secondorder correlation functions $g_{b}^{(2)}(0)$ as functions of the rescaled detuning $\Delta / \kappa$ with different mean thermal photon (phonon) number $n_{\mathrm{th}}$. The equal-time second-order correlation functions $g_{b}^{(2)}(0)$ are plotted as a function of the mean thermal photon (phonon) number $n_{\mathrm{th}}$ in Figs. 5(c) and 5(d).
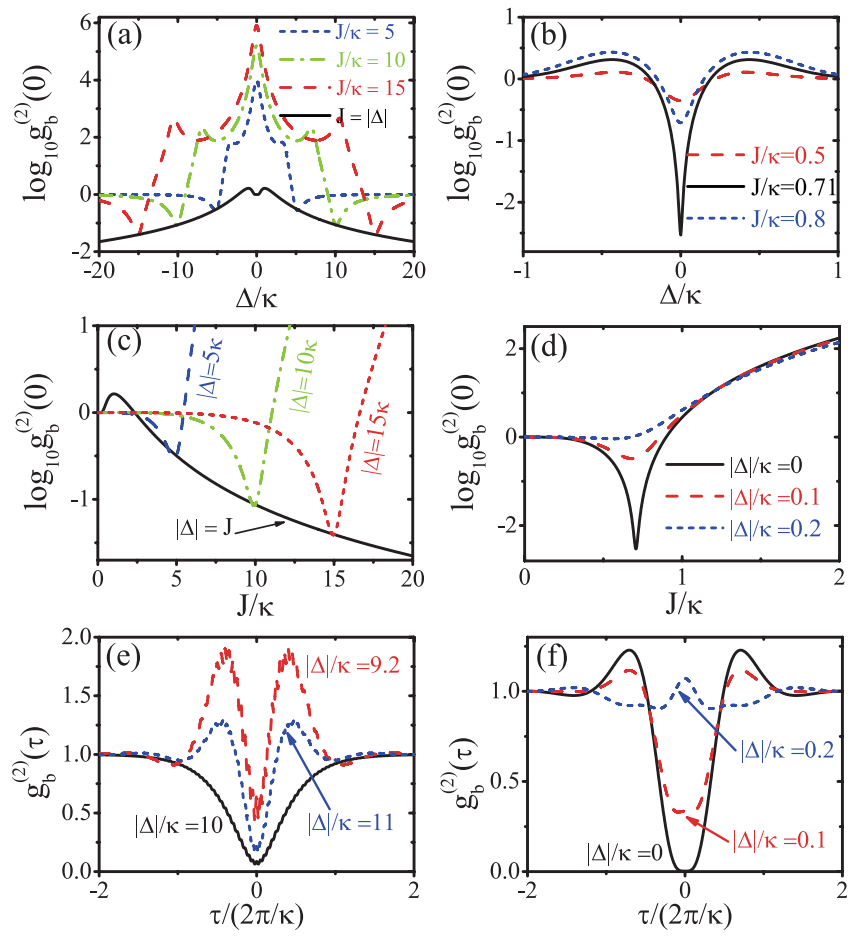

FIG. 3: (Color online) The equal-time second-order correlation function $\log _{10} g_{b}^{(2)}(0)$ is plotted as a function of the detuning $\Delta / \kappa$ in (a) for $J / \kappa$ taking the values $5,10,15,|\Delta| / \kappa$ and in (b) for $J / \kappa$ taking the values $0.5,0.71,0.8$, and as a function of the coupling strength $J / \kappa$ in (c) for $|\Delta| / \kappa$ taking the values $5,10,15, J / \kappa$ and in (d) for $|\Delta| / \kappa$ taking the values $0,0.1,0.2$. The second-order correlation function $g_{b}^{(2)}(\tau)$ is plotted as a function of the normalized time delay $\tau /(2 \pi / \kappa)$ in (e) for $J=10 \kappa$ and $|\Delta| / \kappa$ taking the values 10 , $9.2,11$ and in (f) for $J / \kappa=0.71$ and $|\Delta| / \kappa$ taking the values $0,0.1$, 0.2 . The other parameters are taken as $\gamma=\kappa, \varepsilon=0.01 \kappa, \Omega=0$ and $n_{\mathrm{th}}=0$.

It is clear that the thermal phonons (photons) have a significant effect on both the CPNB and UCPNB under weak driving condition $(\varepsilon=0.01 \kappa)$. The CPNB disappears when the number of the mean thermal phonons reaches $n_{\mathrm{th}}=8.85 \times 10^{-5}$; the UCPNB disappears for the mean number of the thermal phonons $n_{\mathrm{th}}=0.8 \times 10^{-5}$. So that means the UCPNB is more fragile against the thermal noise than the CPNB.

One of the main reasons for the CPNB and UCPNB disappearing in such a small mean thermal photon (phonon) number $\left(n_{\text {th }}<1 \times 10^{-4}\right)$ is that the (total) mean phonon number $n_{b}$ in the NAMR is very small $\left(n_{b} \sim 1 \times 10^{-4}\right)$ for weak external driving $(\varepsilon=0.01 \kappa)$. So in order to improve the robustness against the thermal phonons, we need a larger number of mean phonons in antibunching and one simple way is to enhance the driving strength of the external field $\varepsilon$. The equal-time secondorder correlation functions $g_{b}^{(2)}(0)$ and mean phonon number $n_{b}$ are plotted as functions of the mechanical driving strength $\varepsilon / \kappa$ in Figs. 6(a) and 6(b), respectively. The mean phonon number $n_{b}$ increases as enhancing the driving strength, while the phonons tend to behave classically $\left(g_{b}^{(2)}(0)>1\right)$ when the driving strength becomes strong enough. What's more, we 

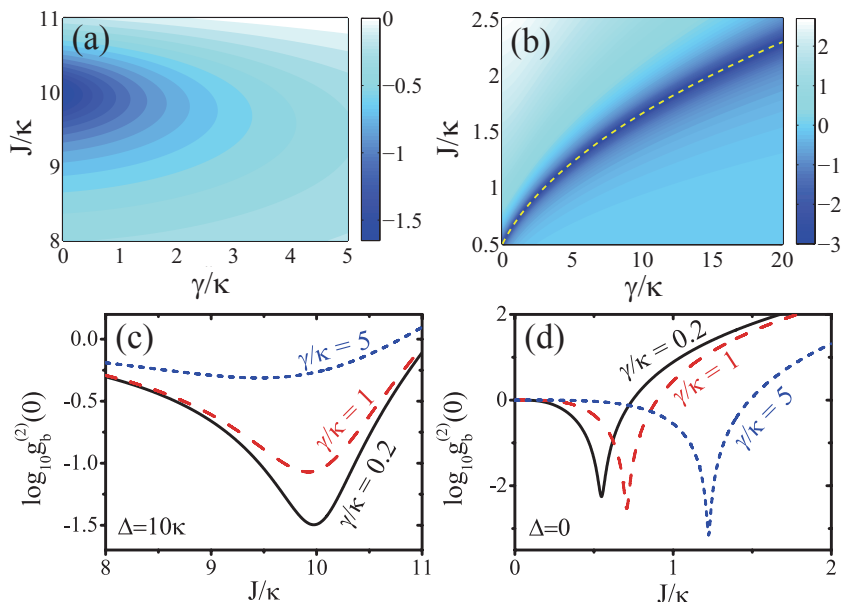

FIG. 4: (Color online) Logarithmic plot of the equal-time secondorder correlation function $\log _{10} g_{b}^{(2)}(0)$ as functions of $\gamma / \kappa$ and $J / \kappa$ in (a) for $\Delta=10 \kappa$ and in (b) for $\Delta=0$. Also $\log _{10} g_{b}^{(2)}(0)$ plotted as a function of $J / \kappa$ in (c) for $\Delta=10 \kappa$ and in (d) for $\Delta=0$ with $\gamma / \kappa=0.2,1$, and 5 . The other parameters are taken as $\varepsilon=0.01 \kappa$, $\Omega=0$ and $n_{\mathrm{th}}=0$. The yellow dash curve in (b) denotes the optimal condition of $J$ given in Eq. (11).
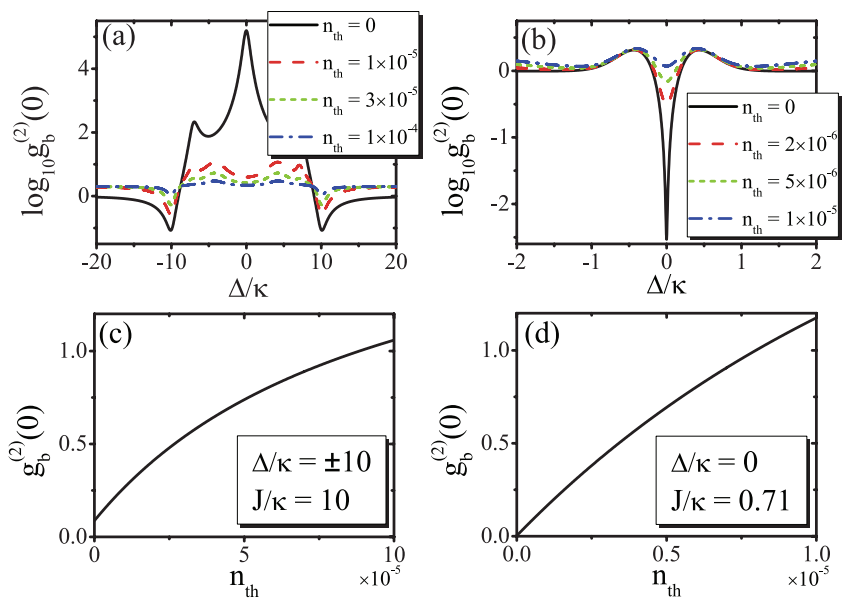

FIG. 5: (Color online) The equal-time second-order correlation functions $\log _{10} g_{b}^{(2)}(0)$ is plotted as a function of the detuning $\Delta / \kappa$ in (a) for $J / \kappa=10$ and in (b) for $J / \kappa=0.71$ with different mean thermal photon (phonon) number $n_{\text {th }}$. The equal-time second-order correlation functions $g_{b}^{(2)}(0)$ is plotted as a function of the mean thermal photon (phonon) number $n_{\mathrm{th}}$ in (c) for $J=|\Delta|=10 / \kappa$ and in (d) for $J / \kappa=0.71$ and $\Delta=0$. The other parameters are taken as $\gamma=\kappa, \varepsilon=0.01 \kappa$, and $\Omega=0$.

can see that the $n_{b}$ in UCPNB is much smaller than the one in CPNB for the same value of $g_{b}^{(2)}(0)$. As shown in Figs. 6(c) and $6(\mathrm{~d})$, the UCPNB can only be observed in much smaller mean number of the thermal phonons than the one for CPNB.

According to the results of Fig. 2 to Fig. 6, we now summarize the properties of CPNB and UCPNB: UCPNB appears in the weak NAMR-qubit coupling condition and the phonon blockade is nearly perfect $\left(g_{b}^{(2)}(0)<0.01\right)$, but UCPNB can
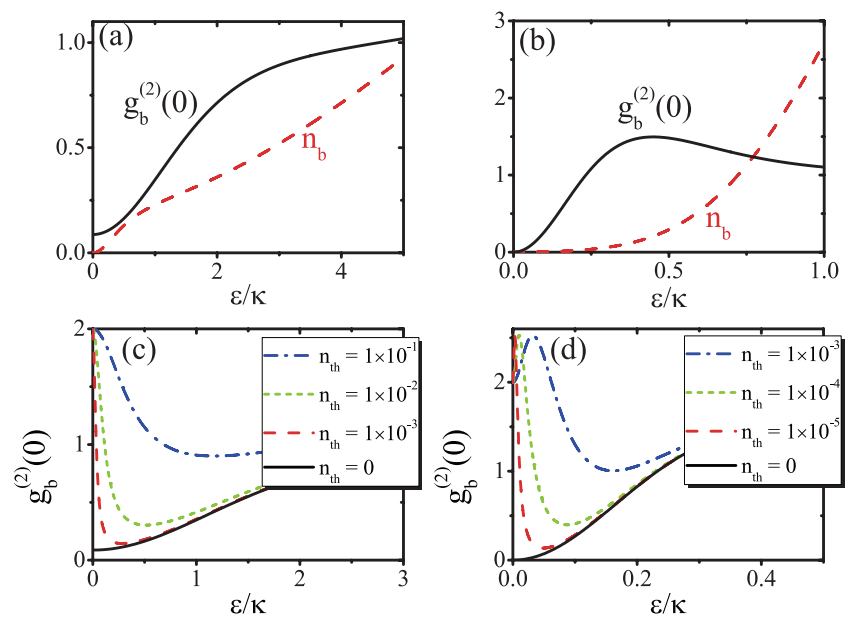

FIG. 6: (Color online) The equal-time second-order correlation functions $g_{b}^{(2)}(0)$ (solid line) and mean phonon number $n_{b}$ (dashed line) are plotted as functions of the mechanical driving strength $\varepsilon / \kappa$ in (a) for $J=|\Delta|=10 / \kappa$ and in (b) for $J / \kappa=0.71$ and $\Delta=0 . g_{b}^{(2)}(0)$ is plotted as a function of the mechanical driving strength $\varepsilon / \kappa$ in (c) for $J=|\Delta|=10 / \kappa$ and in (d) for $J / \kappa=0.71$ and $\Delta=0$ with different thermal photon (phonon) number $n_{\text {th }}$. The other parameters are taken as $\gamma=\kappa, \varepsilon=0.01 \kappa$, and $\Omega=0$.
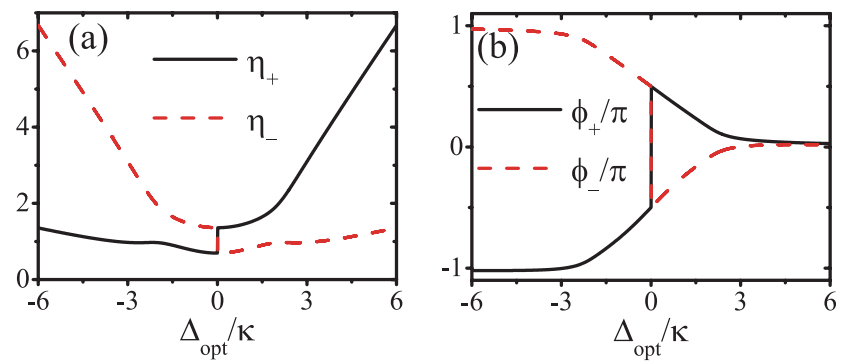

FIG. 7: (Color online) (a) $\eta_{ \pm} / \epsilon$ and (b) $\phi_{ \pm} / \pi$ from Eq. (12) are plotted as functions of the frequency of $\Delta_{\mathrm{opt}} / \kappa$. The other parameters are taken as $\gamma=\kappa$ and $J=J_{\mathrm{opt}}=3 \kappa$.

only be observed with a small mean phonon number and it is very fragile in a reservoir with thermal noise; in contrast to UCPNB, CPNB can be observed with much larger mean phonon number and is more robust against the thermal noise, but CPNB needs very strong NAMR-qubit coupling for strong phonon antibunching. Another reason for us to enlarge the mean phonon number for strong phonon antibunching is that we need to increase the number of single phonons generated by a single-phonon source in a given time.

\section{PHONON BLOCKADE WITH A DRIVING FIELD APPLYING TO THE QUBIT $\Omega \neq 0$}

We now discuss how to enlarge the mean phonon number for strong phonon antibunching with a moderate NAMR-qubit coupling strength $\left(J=J_{\mathrm{opt}}=3 \kappa\right)$ by applying an external driving field to the qubit (i.e., $\Omega \neq 0$ ). As shown in Eq. (12), 

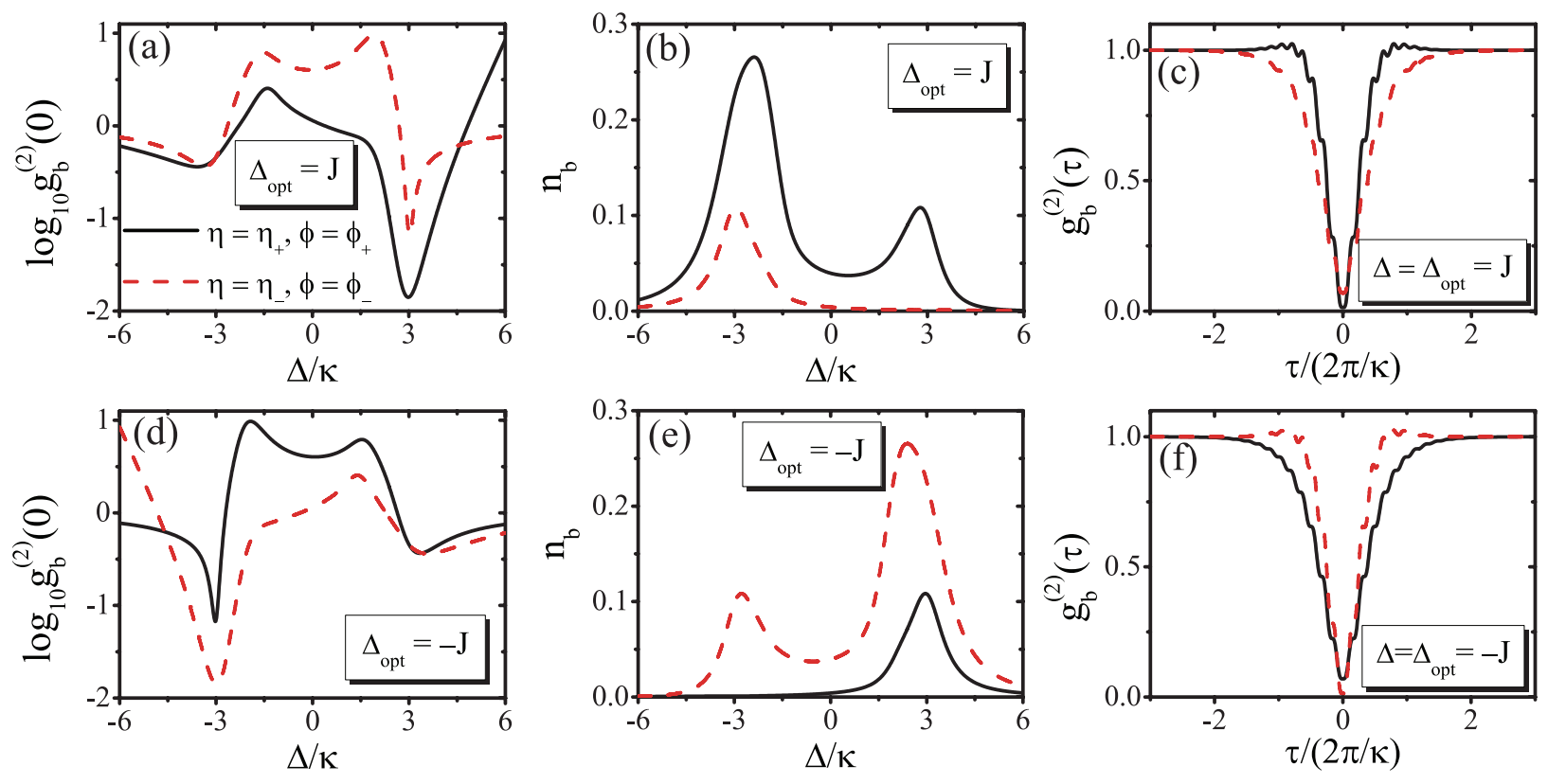

FIG. 9: (Color online) The equal-time second-order correlation functions $\log _{10} g_{b}^{(2)}(0)\left[(\mathrm{a})\right.$ and (d)] and mean phonon number $n_{b}[(\mathrm{~b})$ and (e)] are plotted as functions of the detuning $\Delta / \kappa ; g_{b}^{(2)}(\tau)$ is plotted as a function of the normalized time delay $\tau /(2 \pi / \kappa)$ in (c) and (f) [(a), (b) and (c) for $\Delta_{\mathrm{opt}}=J$; (d), (e) and (f) for $\Delta_{\mathrm{opt}}=-J$ ]. The solid line is plotted for $(\eta, \phi)=\left(\eta_{+}, \phi_{+}\right)$and the dashed line is plotted for $(\eta, \phi)=\left(\eta_{-}, \phi_{-}\right)$. The other parameters are taken as $\gamma=\kappa, \varepsilon=\kappa / 5, J=J_{\mathrm{opt}}=3 \kappa$, and $n_{\mathrm{th}}=0$.
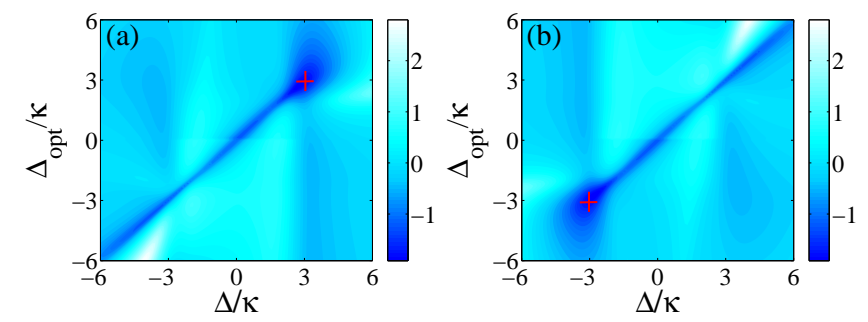

FIG. 8: (Color online) Logarithmic plot of the equal-time secondorder correlation functions $\log _{10} g_{b}^{(2)}(0)$ as functions of the detuning $\Delta / \kappa$ and $\Delta_{\mathrm{opt}} / \kappa$ in (a) for $\eta=\eta_{+}$and $\phi=\phi_{+}$and in (b) for $\eta=\eta_{-}$and $\phi=\phi_{-}$. The red cross marked at (a) $\Delta=\Delta_{\text {opt }} \approx 3 \kappa$ and (b) $\Delta=\Delta_{\mathrm{opt}} \approx-3 \kappa$ denotes the minimal point of $g_{b}^{(2)}(0)$. The other parameters are taken as $\gamma=\kappa, J=J_{\mathrm{opt}}=3 \kappa, \varepsilon=\kappa / 5$, and $n_{\mathrm{th}}=0$.

the optimal parameters ( $\Delta_{\text {opt }}$ and $J_{\text {opt }}$ ) for UCPNB is related to the strength ratio and phase difference $\left(\eta_{ \pm}\right.$and $\left.\phi_{ \pm}\right)$of the

Two snapshots (along the lines of $\Delta_{\mathrm{opt}}= \pm J$ ) taken from Figs. 8(a) and 8(b) are shown in Figs. 9(a) and 9(d), and the corresponding mean phonon number $n_{b}$ is shown in Figs. 9(b) and 9(e). Fig. 9(a) shows that $g_{b}^{(2)}(0)$ for $(\eta, \phi)=\left(\eta_{+}, \phi_{+}\right)$ is smaller than the one for $(\eta, \phi)=\left(\eta_{-}, \phi_{-}\right)$at $\Delta=J=3 \kappa$ when $\Delta_{\mathrm{opt}}=J$; in contrast, when $\Delta_{\mathrm{opt}}=-J$ as shown in Fig. $9(\mathrm{~d}), g_{b}^{(2)}(0)$ for $(\eta, \phi)=\left(\eta_{+}, \phi_{+}\right)$is larger than the one driving fields, so we can combine CPNB and UCPNB together (appear in the same parameter region, i.e., $\Delta_{\text {opt }}= \pm J$ ) by optimizing the values of $\eta_{ \pm}$and $\phi_{ \pm}$. In Fig. 7, parameters $\eta_{ \pm}$ and $\phi_{ \pm} / \pi$ in Eq. (12) are plotted as functions of the detuning of $\Delta_{\text {opt }} / \kappa$. We find that there is an abrupt change of $\eta_{ \pm}$and $\phi_{ \pm}$at $\Delta_{\mathrm{opt}}=0$, and we also find that $\eta_{+}>\eta_{-}$in the region of $\Delta_{\mathrm{opt}}>0$ and $\eta_{+}<\eta_{-}$for $\Delta_{\mathrm{opt}}<0$.

To compare the two solutions in Eq. (12) (optimal conditions) for phonon blockade, the equal-time second-order correlation function $g_{b}^{(2)}(0)$ is shown as a function of the rescaled detunings $\Delta / \kappa$ and $\Delta_{\text {opt }} / \kappa$ in Fig. 8(a) for $(\eta, \phi)=\left(\eta_{+}, \phi_{+}\right)$ and in $8(\mathrm{~b})$ for $(\eta, \phi)=\left(\eta_{-}, \phi_{-}\right)$. Phonons exhibit strong antibunching (blue or dark) in the region around the line $\Delta=\Delta_{\text {opt }}$, and the minimal value of $g_{b}^{(2)}(0)$ appears at $\Delta=$ $\Delta_{\text {opt }} \approx J$ (red cross mark) in Fig. 8(a) for $(\eta, \phi)=\left(\phi_{+}, \eta_{+}\right)$ and at $\Delta=\Delta_{\mathrm{opt}} \approx-J$ (red cross mark) in Fig. 8(b) for $(\eta, \phi)=\left(\phi_{-}, \eta_{-}\right)$.

for $(\eta, \phi)=\left(\eta_{-}, \phi_{-}\right)$at $\Delta=-J=-3 \kappa$. Correspondingly, the mean phonon number $n_{b}$ for $(\eta, \phi)=\left(\eta_{+}, \phi_{+}\right)$ is much larger than the one for $(\eta, \phi)=\left(\eta_{-}, \phi_{-}\right)$at $\Delta=$ $J=3 \kappa$ when $\Delta_{\text {opt }}=J$ as shown in Fig. 9(b); in contrast, $n_{b}$ for $(\eta, \phi)=\left(\eta_{+}, \phi_{+}\right)$is much smaller than the one for $(\eta, \phi)=\left(\eta_{-}, \phi_{-}\right)$at $\Delta=-J=-3 \kappa$ when $\Delta_{\mathrm{opt}}=-J$ as shown in Fig. 9(e). More important, when $(J, \varepsilon)=(3 \kappa, \kappa / 5)$, 


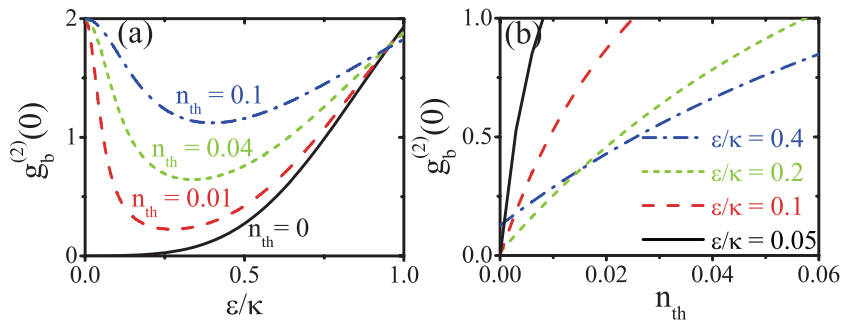

FIG. 10: (Color online) (a) The equal-time second-order correlation functions $g_{b}^{(2)}(0)$ is plotted as a function of the mechanical driving strength $\varepsilon / \kappa$ with different mean thermal photon (phonon) number $n_{\mathrm{th}}$; (b) $g_{b}^{(2)}(0)$ is plotted as a function of the mean thermal photon (phonon) number $n_{\text {th }}$ with different mechanical driving strength $\varepsilon / \kappa$ for $\left(\eta, \phi, \Delta, \Delta_{\mathrm{opt}}\right)=\left(\eta_{+}, \phi_{+}, \Delta_{\mathrm{opt}}, J\right)$ or $\left(\eta, \phi, \Delta, \Delta_{\mathrm{opt}}\right)=$ $\left(\eta_{-}, \phi_{-}, \Delta_{\text {opt }},-J\right)$. The other parameters are taken as $\gamma=\kappa$ and $J=J_{\mathrm{opt}}=3 \kappa$.

the mean phonon number $n_{b}$ can be larger than 0.1 with the optimal parameters $\left(\eta, \phi, \Delta, \Delta_{\text {opt }}\right)=\left(\eta_{+}, \phi_{+}, \Delta_{\text {opt }}, J\right)$ or $\left(\eta, \phi, \Delta, \Delta_{\text {opt }}\right)=\left(\eta_{-}, \phi_{-}, \Delta_{\text {opt }},-J\right)$ for phonon blockade $\left[g_{b}^{(2)}(0) \approx 0.0141\right]$. So in order to obtain a phonon blockade with small $g_{b}^{(2)}(0)$ and large mean phonon number, we should choose the parameters $\left(\eta, \phi, \Delta, \Delta_{\text {opt }}\right)=\left(\eta_{+}, \phi_{+}, \Delta_{\text {opt }}, J\right)$ or $\left(\eta, \phi, \Delta, \Delta_{\mathrm{opt}}\right)=\left(\eta_{-}, \phi_{-}, \Delta_{\mathrm{opt}},-J\right)$. The second-order correlation $g_{b}^{(2)}(\tau)$ is plotted as a function of the normalized time delay $\tau /(2 \pi / \kappa)$ in Figs. 9(c) and 9(f). The time durations of the correlated phonons are about the life time of the phonons in the NAMR.

Next, let us discuss the robustness of the phonon blockade effect against the thermal noise with parameters $\left(\eta, \phi, \Delta, \Delta_{\mathrm{opt}}\right)=\left(\eta_{+}, \phi_{+}, \Delta_{\mathrm{opt}}, J\right)$ or $\left(\eta, \phi, \Delta, \Delta_{\mathrm{opt}}\right)=$ $\left(\eta_{-}, \phi_{-}, \Delta_{\text {opt }},-J\right)$. Figure 10 (a) shows the equal-time second-order correlation functions $g_{b}^{(2)}(0)$ as a function of the mechanical driving strength $\varepsilon / \kappa$ for different mean thermal photon (phonon) number $n_{\text {th }}$, and Fig. 10(b) shows $g_{b}^{(2)}(0)$ as a function of the mean thermal photon (phonon) number $n_{\text {th }}$ for different mechanical driving strength $\varepsilon / \kappa$. From Fig. 10, we can see that the phonon blockade can be observed with the thermal phonon number of 0.06 when $\varepsilon=0.4 \kappa$.

\section{MEASUREMENTS OF PHONON BLOCKADE}

We now turn to study the measurements of phonon blockade in the NAMR-qubit system via an optical cavity field. We assume that the optical cavity field is coupled to the NAMR through the radiation pressure type interaction. We will show that the statistical properties of the phonons in the NAMR can be observed indirectly by measuring photon correlations of the output field from the optical cavity in a similar way as in Refs. $[7,10,30]$. In the rotating reference frame with the frequency of the optical driving field $\omega_{a}$, the Hamiltonian of the total quantum system is given by $(\hbar=1)$

$$
H_{\mathrm{T}}=H_{\mathrm{mq}}+H_{\mathrm{om}},
$$

where

$$
H_{\mathrm{om}}=\Delta_{a} a^{\dagger} a+g a^{\dagger} a\left(b+b^{\dagger}\right)+\left(\Omega_{c} a^{\dagger}+\text { H.c. }\right) .
$$

Here, $a$ and $a^{\dagger}$ denote the annihilation and creation operators of the cavity mode with the frequency $\omega_{c} ; g$ is the vacuum optomechanical coupling strength; $\Omega_{c}$ (assumed to be real) describes the strength of the external driving field which satisfies the resonant condition $\Delta_{a} \equiv \omega_{c}-\omega_{a}=\omega_{m}$. The operator for the cavity mode can be written as the sum of its quantum fluctuation operator and steady-state mean field as $a \rightarrow a+\alpha$, where $\alpha$ is the steady-state mean field at the frequency $\omega_{a}$ of the driving optical field. The optical field at the frequency of the driving optical field will be spectrally filter out and the photon correlation of the quantum fluctuation operator can be measured directly. We note that it is very challenging to measure $g_{a}^{(2)}(\tau)$ of the quantum fluctuation by filtering out $\alpha$. However, the photon counting technique has been realized in a recent experiment [30]. Thus, we will focus on the correlation of the quantum fluctuation operator in the following. In the strong driving condition $|\alpha| \gg 1$, we can linearize the Hamiltonian $H_{\mathrm{om}}$ by only keeping the firstorder terms in the small quantum fluctuation operators, then the linearized Hamiltonian $H_{\mathrm{om}}^{\prime}$ under the rotating wave approximation is given as

$$
H_{\mathrm{om}}^{\prime}=\omega_{m} a^{\dagger} a+G a^{\dagger} b+G^{*} a b^{\dagger},
$$

where the effective optomechanical coupling strength $G=g \alpha$ is assumed to be much smaller than $\omega_{m}$, i.e., $|G| \ll \omega_{m}$.

The quantum Langevin equation for the cavity mode is given by

$$
\frac{d}{d t} a=-\frac{\Gamma}{2} a-i \omega_{m} a-i G b+\sqrt{\Gamma} a_{\mathrm{vac}},
$$

where $\Gamma$ is the damping rate of the cavity mode and the $a_{\mathrm{vac}}$ is the vacuum input noise with the correlation function $\left\langle a_{\mathrm{vac}}^{\dagger}(t) a_{\mathrm{vac}}\left(t^{\prime}\right)\right\rangle=0$. We will focus on the resolved sideband regime $\left(\omega_{m} \gg \Gamma\right)$, and assume that the damping rate $\Gamma$ of the cavity mode is much larger than the effective optomechanical coupling strength $|G|$, the NAMR-qubit coupling strength $J$ and the damping rates of the NAMR and qubit, i.e.,

$$
\omega_{m} \gg \Gamma \gg\left\{|G|, J, \gamma\left(n_{m, \text { th }}+1\right), \kappa\left(n_{q, \text { th }}+1\right)\right\} .
$$

Here, $n_{m, \text { th }}$ and $n_{q, \text { th }}$ denote the mean thermal phonon and photon numbers of the NAMR and qubit, respectively. Then we can obtain the relation between the cavity mode and the NAMR mode [10, 30-32]

$$
a=-i \frac{2 G}{\Gamma} b+f_{\mathrm{vac}}
$$

with the noise term

$$
f_{\mathrm{vac}}=\frac{2}{\sqrt{\Gamma}} \int_{-\infty}^{t}\left[a_{\mathrm{vac}} e^{\left(\frac{\Gamma}{2}+i \omega_{m}\right)(\tau-t)}\right] d \tau .
$$

Since $f_{\text {vac }}$ is the vacuum input, the we have

$$
g_{b}^{(2)}(\tau) \approx g_{a}^{(2)}(\tau)=\frac{\left\langle a^{\dagger}(t) a^{\dagger}(t+\tau) a(t+\tau) a(t)\right\rangle}{\left\langle a^{\dagger}(t) a(t)\right\rangle^{2}} .
$$



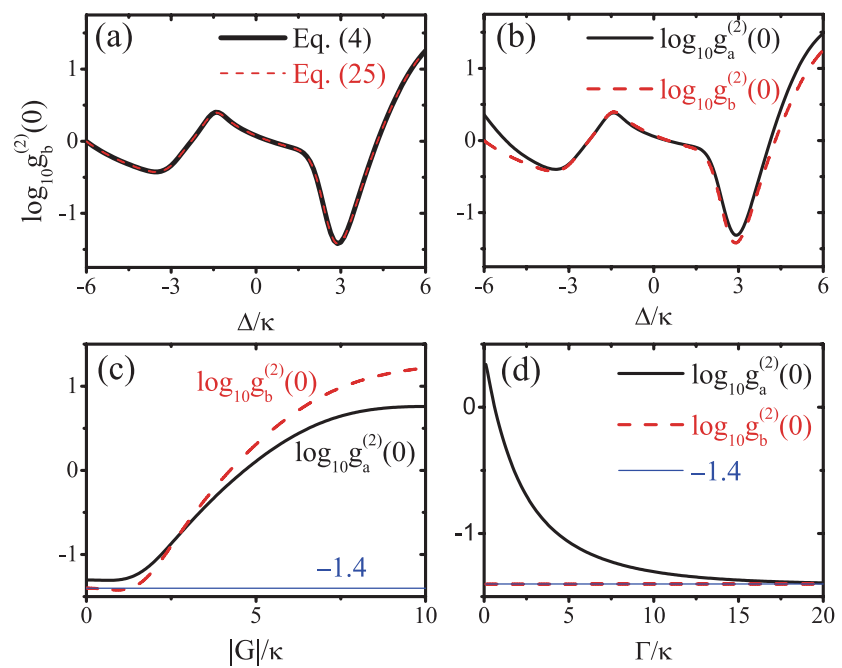

FIG. 11: (Color online) (a) $\log _{10} g_{b}^{(2)}(0)$ is plotted as a function of the detuning $\Delta / \kappa$ by using Eq. (4) (black solid line) and by using Eq. (25) (red dashed line) for $|G|=\kappa / 10$ and $\Gamma=10 \kappa$. $\log _{10} g_{a}^{(2)}(0)$ (black solid line) and $\log _{10} g_{b}^{(2)}(0)$ (red dashed line) are plotted as functions of the detuning $\Delta / \kappa$ for $|G|=\kappa / 10$ and $\Gamma=10 \kappa$ in (b), as functions of $|G| / \kappa$ for $\Gamma=10 \kappa$ and $\Delta=3 \kappa$ in (c) and as functions of $\Gamma / \kappa$ for $|G|=\kappa / 10$ and $\Delta=3 \kappa$ in (d). The blue thin horizontal line for -1.4 in (c) and (d) indicates $\log _{10} g_{b}^{(2)}(0)$ obtained by using Eq. (4). The other parameters are taken as $\left(\eta, \phi, \Delta_{\text {opt }}\right)=\left(\eta_{+}, \phi_{+}, J\right), \gamma=\kappa, \varepsilon=\kappa / 5$, $J=J_{\text {opt }}=3 \kappa$, and $n_{\mathrm{th}}=10^{-3}$.

by using the correlation relation $\left\langle a_{\mathrm{vac}}^{\dagger}(t) a_{\mathrm{vac}}\left(t^{\prime}\right)\right\rangle=0$. Equation (20) shows that phonon blockade in the NAMR can be detected by measuring the photon correlations of the output field from the cavity mode. Note that the effective mechanical damping rate $\widetilde{\gamma}$ and mean thermal phonon number $\widetilde{n}_{m, \text { th }}$ by adiabatically eliminating the optical cavity field under the condition [Eq. (17)] are given by [10, 32-35]

$$
\begin{gathered}
\tilde{\gamma}=\gamma+\gamma_{\mathrm{om}}, \\
\widetilde{n}_{m, \mathrm{th}}=\frac{\gamma n_{m, \mathrm{th}}+\gamma_{\mathrm{om}} n_{\mathrm{om}}}{\gamma+\gamma_{\mathrm{om}}},
\end{gathered}
$$

where

$$
\begin{gathered}
\gamma_{\mathrm{om}}=\frac{4|G|^{2}}{\Gamma} \frac{16 \omega_{m}^{2}}{\Gamma^{2}+16 \omega_{m}^{2}}, \\
n_{\mathrm{om}}=\frac{\Gamma^{2}}{16 \omega_{m}^{2}},
\end{gathered}
$$

are the induced mechanical damping and mean phonon number due to the optomechanical coupling. There is an optomechanically induced frequency shift $\delta \omega=8|G|^{2} \omega_{m} /\left(\Gamma^{2}+\right.$ $\left.16 \omega_{m}^{2}\right)$ of the mechanical resonator, which can be neglected in the condition $|G| \ll \Gamma \ll \omega_{m}$. $g_{a}^{(2)}(0)$ and $g_{b}^{(2)}(0)$ can be obtained by solving the master equation for the total density matrix $\rho_{\mathrm{T}}$ [29]

$$
\begin{aligned}
\frac{\partial \rho_{\mathrm{T}}}{\partial t}= & -i\left[H_{\mathrm{T}}^{\prime}, \rho_{\mathrm{T}}\right]+\Gamma L[a] \rho_{\mathrm{T}} \\
& +\gamma\left(n_{m, \mathrm{th}}+1\right) L[b] \rho_{\mathrm{T}}+\gamma n_{m, \mathrm{th}} L\left[b^{\dagger}\right] \rho_{\mathrm{T}} \\
& +\kappa\left(n_{q, \mathrm{th}}+1\right) L\left[\sigma_{-}\right] \rho_{\mathrm{T}}+\kappa n_{q, \mathrm{th}} L\left[\sigma_{+}\right] \rho_{\mathrm{T}}
\end{aligned}
$$

with the linearized total Hamiltonian $H_{\mathrm{T}}^{\prime}$ in the rotating reference frame with the frequency $\omega_{d}$ of the driving fields as

$$
\begin{aligned}
H_{\mathrm{T}}^{\prime}= & \Delta a^{\dagger} a+\Delta b^{\dagger} b+\Delta \sigma_{+} \sigma_{-} \\
& +G a^{\dagger} b+G^{*} a b^{\dagger}+J\left(\sigma_{+} b+b^{\dagger} \sigma_{-}\right) \\
& +\left(\varepsilon b^{\dagger}+\Omega e^{-i \phi} \sigma_{+}+\text {H.c. }\right)
\end{aligned}
$$

where $L[a] \rho_{\mathrm{T}}$ is the Lindbland term for the optical cavity mode.

In Fig. 11(a), we compare the results of $\log _{10} g_{b}^{(2)}(0)$ calculated by using Eq. (4) (black solid line) and by using Eq. (25) (red dashed line). It clearly shows that they agree well with each other by the parameters $\omega_{m} \gg \Gamma,|G|=\kappa / 10$ and $\Gamma=10 \kappa$. That is because the effect of the optical field is so small $\left(\gamma_{\mathrm{om}} \ll \gamma\right.$ and $\left.\gamma_{\mathrm{om}} n_{\mathrm{om}} \ll \gamma n_{m, \text { th }}\right)$ that we have $\widetilde{\gamma} \approx \gamma$ and $\widetilde{n}_{m, \text { th }} \approx n_{m, \text { th }}$ as given in Eqs. (21) and (22). The correlations of the photons and phonons are shown in Fig. 11(b), which shows $g_{b}^{(2)}(0) \approx g_{a}^{(2)}(0)$ with the same parameters as in Fig. 11(a). So phonon blockade in the NAMR mode can be observed indirectly by measuring photon correlations of the output field from the optical cavity. Moreover, the correlation functions are plotted as functions of $|G| / \kappa$ in Fig. 11(c) and as functions of $\Gamma / \kappa$ in 11(d). We find that when the condition in Eq. (17) is not satisfied (e.g., $\Gamma \sim|G| \gg \kappa$ or $\Gamma \sim|G| \approx \kappa$ ), $\log _{10} g_{a}^{(2)}(0)$ (black solid line) and $\log _{10} g_{b}^{(2)}(0)$ calculated by using Eq. (25) (red dashed line) and Eq. (4) (i.e., -1.4 indicated by blue thin horizontal line) become quite different. These differences can be understood by noting that the influence of the optical field gradually emerges with the increasing of the effective optomechanical coupling strength $|G|$ and the photons in the cavity mode can evolve adiabatically with the phonons in the mechanical mode only when $\Gamma \gg|G| \sim \kappa$.

\section{CONCLUSIONS}

In summary, we have studied phonon blockade in a NAMR which is resonantly coupled to a qubit. We have shown that phonon blockade can be induced not only by strong nonlinear interactions, corresponding to large coupling strengths between the NAMR and the qubit (called as CPNB), but also by the destructive interference between different paths for twophonon excitation with a moderate coupling strength between the NAMR and the qubit (called as UCPNB).

Although UCPNB can appear in a moderate (even weak) NAMR-qubit coupling regime and the phonon blockade is almost perfect, we find that the mean phonon number is very small and it is very fragile in the thermal reservoir. In contrast to UCPNB, CPNB can be observed with much larger mean 
phonon number and more robust against the thermal noise, but it needs very strong NAMR-qubit coupling to blockade subsequent phonons.

We also show that a strong phonon antibunching, with a moderate NAMR-qubit coupling, large mean phonon number and robust against the thermal noise, can be achieved by applying two external driving fields to the NAMR and qubit, respectively. The phonon blockade in the NAMR can be observed indirectly by measuring photon correlations of the output field from the optical cavity which is optomechanically coupled to the NAMR. Our proposal provide a way to observe phonon blockade in the NAMR via its resonant coupling with a qubit.

Finally, let us discuss the experimental feasibility of our proposal. In a recent experiment for a superconducting phase qubit coupled to a NAMR by a Jaynes-Cummings model [1], the NAMR-qubit coupling strength $J / 2 \pi$ is about $124 \mathrm{MHz}$, the frequency of the NAMR $\omega_{m} / 2 \pi$ is about $6 \mathrm{GHz}$, the qubit frequency $\omega_{0} / 2 \pi$ can be set between 5 and $10 \mathrm{GHz}$, the damping rate of the NAMR $\gamma / 2 \pi$ is about $26 \mathrm{MHz}$, the damping rate of the phase qubit $\kappa / 2 \pi$ is about $9 \mathrm{MHz}$, and the environmental temperature can be cooled to $25 \mathrm{mK}$ by a dilution refrigerator. All the parameters used in our paper is within the reach of this experiment [1]. Moreover, in another recent experiment [30], the correlations of phonons for a NAMR in an optomechanical system has been measured by detecting the correlations of the emitted photons from the optical cavity, where the vacuum optomechanical coupling strength $g / 2 \pi$ is $645 \mathrm{kHz}$ and the damping rate of the optical cavity $\Gamma / 2 \pi$ is $817 \mathrm{MHz}$, which satisfy the condition in Eq. (17) for phonon correlation measuring. Thus, if the superconducting NAMR-qubit system can be combined with the optomechanical system as an electro-optomechanical system [36-38], then the phonon blockade in the NAMR should be observed in our proposed system.

\section{Acknowledgement}

X.W.X. thank Yong Li, Hui Wang and Yan-Jun Zhao for fruitful discussions. X.W.X. is supported by the National Natural Science Foundation of China (NSFC) under Grants No.11604096 and the Startup Foundation for Doctors of East China Jiaotong University under Grant No. 26541059. A.X.C. is supported by NSFC under Grant No. 11365009. Y.X.L. is supported by the National Basic Research Program of China(973 Program) under Grant No. 2014CB921401, the Tsinghua University Initiative Scientific Research Program, and the Tsinghua National Laboratory for Information Science and Technology (TNList) Cross-discipline Foundation.

\section{Appendix A: Derivation of optimal equation for UCPNB}

To obtain the optimal conditions for UCPNB in the limit $T \rightarrow 0$, following the method given in Ref. [17], we expand the wave function with the Ansatz

$$
\begin{aligned}
|\psi\rangle= & C_{0, g}|0, g\rangle+C_{0, e}|0, e\rangle+C_{1, g}|1, g\rangle \\
& +C_{1, e}|1, e\rangle+C_{2, g}|2, g\rangle+\cdots .
\end{aligned}
$$

Here, $|n, g\rangle(|n, e\rangle)$ represents that there are $n$ phonons in the mechanical mode and the qubit in the ground (excited) state. The coefficients $\left|C_{n, g}\right|^{2}$ and $\left|C_{n, e}\right|^{2}$ denote occupying probabilities in states $|n, g\rangle$ and $|n, e\rangle$ respectively. Under weak pumping conditions $\left(C_{0, g} \approx 1 \gg\left\{C_{0, e}, C_{1, g}\right\} \gg\right.$ $\left.\left\{C_{1, e}, C_{2, g}\right\} \gg \cdots\right)$, the optimal condition for UCPNB can be obtained for $C_{2, g}=0$.

Substituting the wave function in Eq. (A1) and the Hamiltonian in Eq. (2) into the Schrödinger equation, by taking account of the dampings of the qubit and mechanical mode, we can obtain the dynamical equations for the amplitudes $C_{n, g}$ $\left(C_{n, e}\right)$. The steady-state solutions for amplitudes $C_{1, g}$ and $C_{0, e}$ are determined by

$$
\begin{aligned}
& 0=\left(\Delta-i \frac{\kappa}{2}\right) C_{0, e}+J C_{1, g}+\Omega e^{-i \phi} C_{0, g}, \\
& 0=\left(\Delta-i \frac{\gamma}{2}\right) C_{1, g}+J C_{0, e}+\varepsilon C_{0, g},
\end{aligned}
$$

and the steady-state solutions for amplitudes $C_{2, g}$ and $C_{1, e}$ are determined by

$$
\begin{aligned}
0= & \left(2 \Delta-i \frac{\kappa+\gamma}{2}\right) C_{1, e}+\sqrt{2} J C_{2, g} \\
& +\varepsilon C_{0, e}+\Omega e^{-i \phi} C_{1, g}, \\
0= & (2 \Delta-i \gamma) C_{2, g}+\sqrt{2} J C_{1, e}+\sqrt{2} \varepsilon C_{1, g} .
\end{aligned}
$$

From Eqs. (A2) and (A3), we obtain

$$
\begin{gathered}
C_{0, e}=\frac{\varepsilon J-\Omega\left(\Delta-i \frac{\gamma}{2}\right) e^{-i \phi}}{\left(\Delta-i \frac{\kappa}{2}\right)\left(\Delta-i \frac{\gamma}{2}\right)-J^{2}} C_{0, g}, \\
C_{1, g}=\frac{J \Omega e^{-i \phi}-\varepsilon\left(\Delta-i \frac{\kappa}{2}\right)}{\left(\Delta-i \frac{\kappa}{2}\right)\left(\Delta-i \frac{\gamma}{2}\right)-J^{2}} C_{0, g} .
\end{gathered}
$$

Optimal condition for phonon blockade (i.e., $g_{b}^{(2)}(0) \rightarrow$ 0 ) can be derived by substituting Eqs. (A6) and (A7) into Eqs. (A4) and (A5) with $C_{2, g}=0$, and then we obtain the linear equations for $C_{1, e}$ and $C_{0, g}$

$$
\left(\begin{array}{ll}
M_{11} & M_{12} \\
M_{21} & M_{22}
\end{array}\right)\left(\begin{array}{l}
C_{1, e} \\
C_{0, g}
\end{array}\right)=0
$$

with

$$
\begin{aligned}
M_{11}= & 2 \Delta_{\mathrm{opt}}-i \frac{\kappa+\gamma}{2} \\
M_{12}= & \frac{J_{\mathrm{opt}}}{D}\left(\varepsilon^{2}+\Omega^{2} e^{-i 2 \phi}\right) \\
& -\frac{1}{D}\left(2 \Delta_{\mathrm{opt}}-i \frac{\kappa+\gamma}{2}\right) \varepsilon \Omega e^{-i \phi} \\
M_{21}= & \sqrt{2} J_{\mathrm{opt}} \\
M_{22}= & \frac{\sqrt{2}}{D} J_{\mathrm{opt}} \varepsilon \Omega e^{-i \phi}-\frac{\sqrt{2}}{D}\left(\Delta_{\mathrm{opt}}-i \frac{\kappa}{2}\right) \varepsilon^{2},(\mathrm{~s}) \\
D= & \left(\Delta_{\mathrm{opt}}-i \frac{\kappa}{2}\right)\left(\Delta_{\mathrm{opt}}-i \frac{\gamma}{2}\right)-J_{\mathrm{opt}}^{2},
\end{aligned}
$$


where $\Delta_{\text {opt }}$ and $J_{\mathrm{opt}}$ are the optimal parameters for phonon blockade (i.e., $g_{b}^{(2)}(0) \rightarrow 0$ ) with the corresponding parameters of the external driving fields $(\varepsilon, \Omega$ and $\phi)$. The condition for $C_{1, e}$ and $C_{0, g}$ with nontrivial solutions is that the determinant of the coefficient matrix equals zero, that is

$$
M_{11} M_{22}-M_{12} M_{21}=0 .
$$

We derive the equation for optimal phonon antibunching given in Eq. (5) by simplifying the above equation and obtain a second-order equation in the strength ratio $\eta \equiv \Omega / \varepsilon$ with coefficients $A_{j}(j=1,2,3)$ defined in Eqs. (6)-(8).
[1] A. D. O'Connell, M. Hofheinz, M. Ansmann, R. C. Bialczak, M. Lenander, E. Lucero, M. Neeley, D. Sank, H. Wang, M. Weides, J. Wenner, J. M. Martinis, and A. N. Cleland, Quantum ground state and single-phonon control of a mechanical resonator, Nature (London) 464, 697 (2010).

[2] K. C. Schwab and M. L. Roukes, Putting mechanics into quantum mechanics, Phys. Today 58(7), 36 (2005).

[3] M. A. Kastner, Artificial Atoms, Phys. Today 46(1), 24 (1993).

[4] A. Imamoglu, H. Schmidt, G. Woods, and M. Deutsch, Strongly Interacting Photons in a Nonlinear Cavity, Phys. Rev. Lett. 79, 1467 (1997).

[5] Y. X. Liu, A. Miranowicz, Y. B. Gao, J. Bajer, C. P. Sun, and F. Nori, Qubit-induced phonon blockade as a signature of quantum behavior in nanomechanical resonators, Phys. Rev. A 82, 032101 (2010).

[6] Sh. Barzanjeh and D. Vitali, Phonon Josephson junction with nanomechanical resonators, Phys. Rev. A 93, 033846 (2016).

[7] N. Didier, S. Pugnetti, Y. M. Blanter, and R. Fazio, Detecting phonon blockade with photons, Phys. Rev. B 84, 054503 (2011).

[8] A. Miranowicz, J. Bajer, N. Lambert, Y. X. Liu, and F. Nori, Tunable multiphonon blockade in coupled nanomechanical resonators, Phys. Rev. A 93, 013808 (2016).

[9] X. Wang, A. Miranowicz, H. R. Li, and F. Nori, Method for observing robust and tunable phonon blockade in a nanomechanical resonator coupled to a charge qubit, Phys. Rev. A 93, 063861 (2016).

[10] T. Ramos, V. Sudhir, K. Stannigel, P. Zoller, and T. J. Kippenberg, Nonlinear Quantum Optomechanics via Individual Intrinsic Two-Level Defects, Phys. Rev. Lett. 110, 193602 (2013).

[11] H. Wang, X. Gu, Y. X. Liu, A. Miranowicz, and F. Nori, Tunable photon blockade in a hybrid system consisting of an optomechanical device coupled to a two-level system, Phys. Rev. A 92, 033806 (2015).

[12] K. M. Birnbaum, A. Boca, R. Miller, A. D. Boozer, T. E. Northup, and H. J. Kimble, Photon blockade in an optical cavity with one trapped atom, Nature (London) 436, 87 (2005).

[13] A. Faraon, I. Fushman, D. Englund, N. Stoltz, P. Petro, and J. Vuckovic, Coherent generation of non-classical light on a chip via photon-induced tunnelling and blockade, Nat. Phys. 4, 859 (2008).

[14] C. Lang, D. Bozyigit, C. Eichler, L. Steffen, J. M. Fink, A. A. Abdumalikov, Jr., M. Baur, S. Filipp, M. P. da Silva, A. Blais, and A. Wallraff, Observation of Resonant Photon Blockade at Microwave Frequencies Using Correlation Function Measurements, Phys. Rev. Lett. 106, 243601 (2011).

[15] A. J. Hoffman, S. J. Srinivasan, S. Schmidt, L. Spietz, J. Aumentado, H. E. Tureci, and A. A. Houck, Dispersive Photon Blockade in a Superconducting Circuit, Phys. Rev. Lett. 107, 053602 (2011).

[16] T. C. H. Liew and V. Savona, Single Photons from Coupled Quantum Modes, Phys. Rev. Lett. 104, 183601 (2010).
[17] M. Bamba, A. Imamoglu, I. Carusotto, and C. Ciuti, Origin of strong photon antibunching in weakly nonlinear photonic molecules, Phys. Rev. A 83, 021802(R) (2011).

[18] M.-A. Lemonde, N. Didier, and A. A. Clerk, Antibunching and unconventional photon blockade with Gaussian squeezed states, Phys. Rev. A 90, 063824 (2014).

[19] A. Majumdar, M. Bajcsy, A. Rundquist, and J. Vuckovic, Loss-Enabled Sub-Poissonian Light Generation in a Bimodal Nanocavity, Phys. Rev. Lett. 108, 183601 (2012).

[20] P. Kómár, S. D. Bennett, K. Stannigel, S. J. M. Habraken, P. Rabl, P. Zoller, and M. D. Lukin, Single-photon nonlinearities in two-mode optomechanics, Phys. Rev. A 87, 013839 (2013).

[21] D. Gerace and V. Savona, Unconventional photon blockade in doubly resonant microcavities with second-order nonlinearity, Phys. Rev. A 89, 031803(R) (2014).

[22] O. Kyriienko, I. A. Shelykh, and T. C. H. Liew, Tunable singlephoton emission from dipolaritons, Phys. Rev. A 90, 033807 (2014).

[23] X. W. Xu and Y. Li, Strong photon antibunching of symmetric and antisymmetric modes in weakly nonlinear photonic molecules, Phys. Rev. A 90, 033809 (2014).

[24] X. W. Xu and Y. Li, Tunable photon statistics in weakly nonlinear photonic molecules, Phys. Rev. A 90, 043822 (2014).

[25] O. Kyriienko and T. C. H. Liew, Triggered single-photon emitters based on stimulated parametric scattering in weakly nonlinear systems, Phys. Rev. A 90, 063805 (2014).

[26] H. Z. Shen, Y. H. Zhou, and X. X. Yi, Tunable photon blockade in coupled semiconductor cavities, Phys. Rev. A 91, 063808 (2015).

[27] Y. H. Zhou, H. Z. Shen, and X. X. Yi, Unconventional photon blockade with second-order nonlinearity, Phys. Rev. A 92, 023838 (2015).

[28] J. Tang, W. Geng, and X. Xu, Quantum Interference Induced Photon Blockade in a Coupled Single Quantum Dot-Cavity System, Sci. Rep. 5, 9252 (2015).

[29] H. J. Carmichael, An Open Systems Approach to Quantum Optics, Lecture Notes in Physics Vol. 18 (Springer-Verlag, Berlin, 1993).

[30] J. D. Cohen, S. M. Meenehan, G. S. MacCabe, S. Groblacher, A. H. Safavi-Naeini, F. Marsili, M. D. Shaw, and O. Painter, Phonon counting and intensity interferometry of a nanomechanical resonator, Nature (London) 520, 522 (2015).

[31] K. Jahne, C. Genes, K. Hammerer, M. Wallquist, E. S. Polzik, and P. Zoller, Cavity-assisted squeezing of a mechanical oscillator, Phys. Rev. A 79, 063819 (2009).

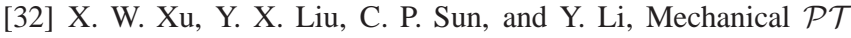
symmetry in coupled optomechanical systems, Phys. Rev. A 92, 013852 (2015).

[33] I. Wilson-Rae, N. Nooshi, W. Zwerger, and T. J. Kippenberg, Theory of Ground State Cooling of a Mechanical Oscillator Using Dynamical Backaction, Phys. Rev. Lett. 99, 093901 (2007).

[34] F. Marquardt, J. P. Chen, A. A. Clerk, and S. M. Girvin, Quan- 
tum Theory of Cavity-Assisted Sideband Cooling of Mechanical Motion, Phys. Rev. Lett. 99, 093902 (2007).

[35] Y. Li, Y. D. Wang, F. Xue, and C. Bruder, Quantum theory of transmission line resonator-assisted cooling of a micromechanical resonator, Phys. Rev. B 78, 134301 (2008).

[36] J. Bochmann, A. Vainsencher, D. D. Awschalom, and A. N. Cleland, Nanomechanical coupling between microwave and optical photons, Nature Phys. 9, 712 (2013).

[37] T. Bagci, A. Simonsen, S. Schmid, L. G. Villanueva, E.
Zeuthen, J. Appel, J. M. Taylor, A. Sørensen, K.Usami, A. Schliesser, and E. S. Polzik, Optical detection of radio waves through a nanomechanical transducer, Nature (London) 507, 81 (2014).

[38] R. W. Andrews, R. W. Peterson, T. P. Purdy, K. Cicak, R. W. Simmonds, C. A. Regal, and K. W. Lehnert, Bidirectional and efficient conversion between microwave and optical light, Nature Phys. 10, 321 (2014). 